$$
y=-\int_{a}^{b} G(x, \xi) p(\xi) d \xi .
$$

A special case deserves mention on account of its simplicity, viz., the case where conditions $(b)$ reduced to

$$
G(a, \xi)=G^{\prime}(a, \xi)=\cdots=G^{[k-1]}(a, \xi)=0
$$

and conditions $(c)$ to

$$
G(b, \xi)=G^{\prime}(b, \xi)=\cdots=G^{[n-k-1]}(b, \xi)=0,
$$

accents denoting differentiation with regard to $x$. One of the simplifications introduced by this specialization is that when $x$ and $\xi$ are now interchanged, the effect is to interchange $a$ and $b$ in conditions $(b)$ and $(c)$. It will be seen that for a given equation (1) and a given interval $(J)$ there are $n-1$ Green's functions of this special sort, obtained by giving to $k$ the values $1,2, \cdots, n-1$. These functions might properly be called Green's functions of the first kind, since when $n=2$ there is only one of them, namely the Green's function, which vanishes at both ends of $(J)$.

Göttingen, Germany, February 8, 1901.

\title{
ON A SYSTEM OF PLANE CURVES HAVING FACTORABLE PARALLELS.
}

BY DR. VIRGIL SNYDER.

(Read before the American Mathematical Society, December 28, 1900.)

The type of scrolls contained in a linear congruence, and having factorable asymptotic lines, gives rise to a class of plane curves whose parallels have a similar property.

It has been shown * that the spherical images of such scrolls have factorable lines of curvature and a method was given by means of which a scroll of this type could be transformed into an annular surface having only plane lines of curvature, the curve of each section breaking up into two factors. The planes of the system all belong to the same axial pencil, and the locus of centers of the generating spheres lies in the bisecting plane of the angle between the two plane directors.

*V. Snyder, "On a special form of annular surface." Amer. Jour. of Math., vol. 23 . 
When the axis of this pencil of planes lies in the plane at infinity, the director planes become antiparallel, the generating spheres have a constant radius, and their envelope is a tubular surface. The procedure is as follows : take any two parallel lines

$$
\text { (q) } y=x t, z=0 ; \quad \text { (p) } y+x t=0, z=0 ;
$$

these lines go over into two parallel planes in sphere space. Take the conjugate of $(p)$ with regard to the complex $c$, image of point spheres. 'This line $\left(p^{\prime}\right)$ is $x=x z, t=0$.

The image of this line (which does not cut $(q)$ ) is the same plane as that of $p$, but now regarded as generated by its second pencil of minimum lines, so that the direction of the normal is reversed. Any scroll having these two lines $\left(p^{\prime}\right)$ and $(q)$ for directrices will be defined by

$$
\frac{y-x t}{z}=\lambda, \quad \frac{x-x z}{t}=\mu,
$$

the relation between $\lambda$, $\mu$ being determined by the third director curve $f(\lambda, \mu)=0$.

The spherical image of this scroll has for its equation

$$
\begin{aligned}
& \text { (1) } \quad\left|\begin{array}{cccc}
\tau & 0 & \zeta & -(\xi+i \eta) \\
0 & \tau & -(\xi-i \eta) & -\zeta \\
0 & 1 & -\lambda & -x \\
1 & 0 & -x & -\mu
\end{array}\right|=0 \\
& \text { or } \quad\left(\xi-\frac{(\mu+\lambda) \tau}{2}\right)^{2}+\left(\eta-\frac{(\lambda-\mu) i \tau}{2}\right)^{2}+\zeta^{2}=x^{2} \tau^{2},
\end{aligned}
$$

$i$. e., it is the envelope of a sphere of constant radius $x$, whose center moves in the plane $\zeta=0$, subject to the law $f(\lambda, \mu)=0$. In particular, if $f(\lambda, \mu)=0$ defines a scroll of the type mentioned, the lines of curvature of the second system belonging to this tube will be factorable. But it is at once evident that these curves are simply the parallels of the locus of centers.

Conversely, if such a tube is generated from a curve whose parallels are known to be factorable, the general annular surface derived from it and also the scroll, its line space image, will have the same property. Starting from this space, let $\varphi(\alpha, \beta)=0$ be the equation of any plane curve; then

$$
(\xi-(m \beta+\alpha))^{2}+\eta^{2}+\zeta^{2}=\beta^{2}+m^{2} \xi^{2}, \quad m=\frac{d \beta}{d \alpha},
$$


is the equation of a sphere whose envelope is the surface of revolution formed by rotating $\varphi(\alpha, \beta) \equiv \varphi(\xi, \eta)=0$ about the $\xi$-axis. This surface is an annular one contained in a linear congruence, its two director planes being $\zeta \pm i \eta=0$. The image in line space of this sphere is

$$
\begin{aligned}
\beta \sqrt{1+m^{2}} z & =x-(m \beta+\alpha) t, \\
(m \beta+\alpha) z & =y-\beta \sqrt{1+m^{2} t} ;
\end{aligned}
$$

and the images of the directors are as follows:

$$
\begin{aligned}
& \zeta+i \eta=0 \text { becomes } z+t=0, x+y=0, \\
& \zeta-i \eta=0 \quad \text { " } \quad z=t, \quad x=y .
\end{aligned}
$$

The generators of the scroll having these two lines for directrices must be of the form

$$
\frac{x+y}{z+t}=\lambda, \quad \frac{x-y}{z-t}=\lambda
$$

making them identical with the above form, we have

$$
\frac{\mu+\lambda}{2} z=x+\frac{(\lambda-\mu)}{2} t, \frac{(\mu-\lambda)}{2} z=y-\frac{(\lambda+\mu)}{2} t
$$

from which

$$
\frac{\mu+\lambda}{2}=\beta \sqrt{1+m^{2},} \quad \frac{\mu-\lambda}{2}=m \beta+\alpha .
$$

But when the directrices are transformed, the meaning of $\lambda, \mu$ remains the same and equation (1) shows what these directrices give when so transformed that the spherical image shall be a tubular surface. Hence : the equations

$$
\begin{gathered}
x=\beta \sqrt{1+\left(\begin{array}{l}
d \beta \\
d \alpha
\end{array}\right)^{2}}, \quad y=i\left(\alpha+\beta \frac{d \beta_{i}}{d \alpha}\right) \\
\varphi(\alpha, \beta)=0, \quad \varphi(\alpha, \beta) \neq \varphi(\alpha,-\beta)
\end{gathered}
$$

define a curve whose parallel curves break up into two distinct factors, corresponding to the positive and negative directions of the normal, $i$. e., toward or away from the center of curvature from the point on the curve.

It is not difficult to generate other surfaces, all of whose lines of curvature of both systems are plane, and those of one system factorable; any "surface moulure" having a factorable director curve is of this type, * but the above

* See Enneper, "Untersuchungen über die Flächen mit planen und sphärischen Krümmungslinien," Göttinger Abhandl., vol. 23, cf. p. 45, eq. (68); and Bianchi, Differentialgeometrie, ch. 5, \& 74. 
surfaces are the only annular surfaces of the class. The second mantle of the surface of centers is the cylinder erected on the evolute of the locus of centers of the generating spheres. When this cylinder becomes a straight line the surface is a torus.

The factorability of the asymptotic lines of a scroll contained in a linear congruence depends entirely upon the configuration at the pinch points, all of which are situated on the linear directrices. When such a scroll is transformed into a tubular surface, these points go into the circular points of the plane containing the locus of centers; hence the configuration of the curve at the circular points determines whether its parallels be factorable or not.

In particular, when the equation of the scroll is of the form

$$
\mu \varphi(\lambda)+f(\lambda)=0
$$

the asymptotic lines are factorable when

$$
\left|\begin{array}{cc}
\varphi & f \\
\varphi^{\prime} & f^{\prime}
\end{array}\right| \neq 0
$$

is a perfect square (or a constant); but this is the condition that all of the pinch points are double, so that the $n$ generators from each point of the multiple directrix are always the same, $i . e ., x$ real, $n-x$ imaginary, $x$ being constant.

Scrolls of this type exist for every value of $n$.

The corresponding condition for the curve is that $\alpha^{2}+\beta^{2}$ should be equal to a square (or a constant), $\alpha^{2}+\beta^{2}$ being the tangential equation of the circular points. For curves of class 2 or 4 this expresses that the foci of its dual curve must all be singular, but this is not true when the class is higher. Thus the dual of the general bicircular quartic has not factorable parallels, but those of the cartesian ovals, limaçon, cardioid, etc., have-the latter giving the cubic, $a\left(x^{2}+y^{2}\right)=(a+2 x)^{3}$.

This equation $\varphi^{2}(\alpha, \beta, \gamma)\left(\alpha^{2}+\beta^{2}\right)=f^{2}(\alpha, \beta, \gamma)$ is of more general application than to the $(n, 1)$ scrolls and seems necessary and sufficient in every case.*

\footnotetext{
* This equation is derived in a different way in Salmon's Higher plane curves, 3d edition, p. 103.

CoRNeLL UNIVERSITY, October 30, 1900.
} 